\title{
Schizophrenia severity, social functioning and hippocampal neuroanatomy: three-dimensional mapping study
}

\author{
P. Brambilla, C. Perlini, P. Rajagopalan, P. Saharan, G. Rambaldelli, M. Bellani, N. Dusi, \\ R. Cerini, R. Pozzi Mucelli, M. Tansella and P. M. Thompson
}

\section{Background}

Hippocampal shrinkage is commonly reported in schizophrenia, but its role in the illness is still poorly understood. In particular, it is unclear how clinical and psychosocial variables relate to hippocampal volumes.

\section{Aims \\ To investigate neuroanatomic differences in the hippocampus using three-dimensional (3D) computational image analysis.}

\section{Method}

We used high-resolution magnetic resonance imaging and surface-based modelling to map the 3D profile of hippocampal differences in adults with schizophrenia $(n=67)$ and a healthy control group $(n=72)$. Manual tracings were used to create 3D parametric mesh models of the hippocampus. Regression models were used to relate diagnostic measures to maps of radial distance, and colourcoded maps were generated to show the profile of associations.

\section{Results}

There was no detectable difference between the schizophrenia and control groups in hippocampal radial distance. In the schizophrenia group, however, bilateral shape deflation was associated with greater illness severity (length of illness, positive and negative symptoms) and with poorer social functioning (educational level, quality of life and health status), which survived Bonferroni correction.

\section{Conclusions}

IIIness severity and poor social functioning may be associated with hippocampal deflation in schizophrenia. As a structural sign of poor outcome, imaging measures might help to identify a subgroup of patients who may need specific treatment to resist hippocampal shrinkage, such as cognitive rehabilitation or physical exercise.

\section{Declaration of interest}

None.

From these findings it is not yet fully understood whether the Conventional volumetric magnetic resonance imaging (MRI) studies suggest that at least a proportion of people with schizophrenia, particularly men and those with a first psychotic episode, ${ }^{1,2}$ have smaller hippocampal volumes than controls, as shown by systematic review and meta-analysis of regional morphometry. ${ }^{3,4}$ Decreased hippocampal size may be accompanied by reductions in $\mathrm{N}$-acetylaspartate levels, ${ }^{5}$ and by hyperactivity of excitatory cortical pathways. ${ }^{6}$ One meta-analysis reported slightly lower hippocampal volumes in non-psychotic first-degree relatives of patients with schizophrenia. The effect size was small to moderate, and not consistently found - especially for the right hippocampus - making the results hard to interpret.? Interestingly, a study investigating people at high risk of psychosis, patients with first-episode psychosis and individuals with chronic schizophrenia found hippocampal structural differences after the onset of the disease, ${ }^{8}$ as also confirmed by a meta-analysis of voxel-based structural MRI studies. ${ }^{9}$ The same group showed, counterintuitively, that larger rather than smaller hippocampal volumes in the high-risk cohort predicted subsequent development of acute psychosis, i.e. people who later developed a psychotic disorder had larger volumes than the high-risk non-psychotic subgroup, with no difference from the normal group. ${ }^{10}$ In addition, prior findings in schizophrenia have been inconsistent, reporting preserved volumes, ${ }^{11-14}$ as also shown by a voxel-wise meta-analysis using anatomic likelihood estimation to identify consistently implicated brain areas across studies, ${ }^{15}$ or minimal volume reductions of about $4 \% .^{16}$ In this regard, in our prior region of interest study we found no hippocampal volume abnormality in schizophrenia. ${ }^{17}$ Some post-mortem studies agree with imaging findings showing hippocampal atrophy in schizophrenia, ${ }^{18,19}$ but others report no neuropathological abnormality. ${ }^{20,21}$ hippocampus is consistently altered in schizophrenia; however, at least during the onset phase of the disease, dynamic processes in the brain may change its morphology. It is indeed a plastic structure sensitive to environmental, clinical and psychosocial phenomena. As such, it is of interest to investigate further whether potential hippocampal changes reflect neurodevelopmental anomalies, an aspect of disease progression or structural sequelae of psychosocial changes that accompany the illness. The role of such dimensions in affecting, or associating with, hippocampus volumes in schizophrenia has been underinvestigated. To this extent, schizophrenia is a heterogeneous syndrome including people with a relatively positive course of illness and others with a far poorer long-term prognosis. One strategy for better understanding the pathophysiology of the disease would be to relate specific clinical and social variables to deviations from normal brain anatomy. In this context different outcome measures have been used, such as duration and severity of positive symptoms, recovery from the first episode, psychopathological indicators of the Brief Psychiatric Rating Scale or functioning as detected with the Global Assessment of Functioning scale (see Bellani et al for a review). ${ }^{22}$

We report results of the first study (to our knowledge) to use three-dimensional (3D) surface mesh models to evaluate statistical effects of clinical and psychosocial variables on the structural anatomy of the hippocampus in schizophrenia. This advanced computational imaging technique examines, at high spatial resolution, neuroanatomical hippocampal differences with more spatial detail than conventional manual tracing methods, allowing calculation of the local radial shrinkage. ${ }^{23}$ It can detect subtle or localised alterations in hippocampal structure, even when 
differences in overall hippocampal volumes are not detectable, as in our prior study. ${ }^{17}$

\section{Method}

A total of 67 patients with a DSM-IV diagnosis of schizophrenia and a control group of 72 healthy individuals were studied (see Table 1). ${ }^{24}$ Patients were recruited from the geographically defined catchment area of South Verona (around 100000 inhabitants). They were being treated by the South Verona community-based mental health service and by other clinics reporting to the South Verona Psychiatric Care Register. Diagnoses of schizophrenia were obtained using the Item Group Checklist of the Schedule for Clinical Assessment in Neuropsychiatry (IGC-SCAN), ${ }^{25}$ administered by two trained research clinical psychologists with extensive experience in this measure; they had already administered at least ten previous IGC-SCAN assessments with a trained senior investigator. The Italian version of the SCAN was used, edited by our group. ${ }^{26}$ The psychopathological item groups completed by the two raters were compared, to discuss any discrepancies in the assessment of major symptoms. Diagnoses of schizophrenia were corroborated by the clinical consensus of two staff psychiatrists according to DSM-IV criteria. We also ensured the reliability of the IGC-SCAN diagnoses by holding regular consensus meetings with the psychiatrists treating the patients and a senior investigator. Patients with comorbid psychiatric disorders, alcohol or substance misuse within the 6 months preceding the study, history of traumatic head injury with loss of consciousness, epilepsy or other neurological disease were excluded. The patients' antipsychotic medication was recorded and chlorpromazine equivalent dosages were calculated (Table 1). Patients' clinical information was retrieved from psychiatric interviews, the attending psychiatrist and medical charts. Clinical symptoms were characterised using the 24-item Brief Psychiatric Rating Scale (BPRS) and psychosocial functioning with the 36-item Short Form Health Questionnaire (SF-36) and the Manchester Short Assessment of Quality of Life (MANSA). ${ }^{27-29}$ These scales were administered by two trained clinical research psychologists. The reliability of the BPRS was established and monitored using procedures similar to those for the IGC-SCAN.

Participants in the control group had no DSM-IV Axis I disorder, determined using a brief modified version of the Structured Clinical Interview for DSM-IV - Non-Patient Version, ${ }^{30}$ no history of psychiatric disorder among first-degree relatives, no history of alcohol or substance misuse and no current major medical illness. All participants gave signed informed consent, after an explanation of all issues involved in participation in the research. The research was approved by the biomedical ethics committee of the Azienda Ospedaliera of Verona.

\section{Psychosocial scales}

MANSA

The MANSA is a clinical interview originally developed as a brief modified version of the Lancashire Quality of Life Profile. ${ }^{29}$ The scale encompasses three sections: the first describes stable personal patient details, such as personal information and diagnosis; the second records personal details that may change over time, such as education, employment, income, accommodation and family members living with the patient; and the third section consists of 16 questions that must be asked every time the assessment is applied. In this section, 4 questions address objective items (having a close friend, number of contacts with friends per week, judicial proceedings and physical violence experienced) and require a 'yes' or 'no' answer; the remaining 12 questions address subjective domains (satisfaction with life, employment or training, financial situation, quality of friendships, hobbies, satisfaction with accommodation, personal safety, sexual life, relationship with family, physical and mental health) and are rated on a seven-point scale, from 'extremely negative' (score 1) to 'extremely positive' (score 7).

\section{SF-36}

The SF-36 is one of the most widely used generic health status measures. ${ }^{28}$ It is composed of 36 questions and standardised response choices, divided into eight multi-item scales: physical functioning, role limitations due to physical health problems, bodily pain, general health perceptions, vitality, social functioning, role limitation due to emotional problems, and general mental health. It is applied to assess the previous 4 weeks.

\section{Image acquisition}

Magnetic resonance imaging scans were acquired with a $1.5 \mathrm{~T}$ Siemens Magnetom Symphony Maestro Class scanner, Syngo MR, 2002B (Siemens, Erlangen, Germany). A standard head coil was used for radio-frequency transmission and reception of the magnetic resonance signal; restraining foam pads were used to minimise head motion. First, $T_{1}$-weighted images were obtained to verify each participant's head position and the image quality, with acquisition parameters repetition time (TR) $450 \mathrm{~ms}$, time to echo (TE) $14 \mathrm{~ms}$, flip angle $90^{\circ}$, field of view (FOV) $230 \times 230 \mathrm{~mm}^{2}, 18$ slices, slice thickness $5 \mathrm{~mm}$, matrix size $384 \times 512$, number of excitations (NEX) 2. Proton density and $T_{2}$-weighted images were then acquired $(\mathrm{TR}=2500 \mathrm{~ms}$, $\mathrm{TE}=24 / 121 \mathrm{~ms}$, flip angle $180^{\circ}, \mathrm{FOV}=230 \times 230 \mathrm{~mm}^{2}, 20$ slices, slice thickness $5 \mathrm{~mm}$, matrix size $410 \times 512$, NEX 2) according to an axial plane parallel to the anterior-posterior commissures to exclude focal lesions. Subsequently, a coronal 3D magnetisation prepared rapid gradient echo (MP-RAGE) sequence was acquired $\left(\mathrm{TR}=2060 \mathrm{~ms}, \mathrm{TE}=3.9 \mathrm{~ms}\right.$, flip angle $15^{\circ}, \mathrm{FOV}=176 \times 235 \mathrm{~mm}^{2}$, slice thickness $1.25 \mathrm{~mm}$, matrix size $270 \times 512$, inversion time $1100 \mathrm{~ms}$ ) to obtain 144 images covering the entire brain.

\section{Image processing and analysis}

Anatomical imaging data were transferred to a personal computer workstation and analysed using the BRAINS2 software developed at the University of Iowa (http://www.nitrc.org/search/?type-ofsearch $=$ group $\&$ cat $=498$ :BRAINS+License). The hippocampus was manually traced on the $T_{1}$-weighted (MP-RAGE) images in the coronal plane as previously described by our group. ${ }^{31}$ Specifically, the corona radiata and the ambient cistern were used as the superior border, the white matter served as the inferior border and the inferior horn of the lateral ventricle as the lateral one. The parahippocampal gyrus and other surrounding areas, such as entorhinal and perirhinal regions, were not included in the tracing. The hippocampus for this study was rigidly aligned and re-sliced for tracing, i.e. only a rigid body transform was used to align the brains for tracing, to avoid re-scaling the anatomy. Intracranial volume was traced in the coronal plane along the border of the brain and included the cerebrospinal fluid, dura mater, sinus, optic chiasm, brainstem, cerebral and cerebellar matter. The inferior border did not extend below the base of the cerebellum. The first and last ten slices including brain matter were traced, then one slice in every five was traced. Tracing was performed by two raters masked to the participant's identity and to sociodemographic and clinical variables. They achieved high reliability, as defined by intraclass correlation coefficients of 0.96 for both left and right hippocampal volumes and 0.97 for 
intracranial volume, obtained by masking on ten scans. The volumes were obtained by summing the volumes of all relevant slices and were expressed in ml. Raw measures were obtained.

\section{Statistical analysis}

Three-dimensional parametric surface maps were created from the automatically generated segmentations of each individual's hippocampus using anatomical surface modelling software. ${ }^{23} \mathrm{~A}$ 3D medial curve was calculated from the hippocampal traces, computed separately for each individual. Local radial shape deflation was calculated by measuring the radial distances from each hippocampal surface point on the 3D surface mesh to the medial axis. Based on computed point-wise correspondences, surface models for all individuals in each diagnostic group were geometrically averaged. Statistical maps indicating local volumetric differences between groups were generated from the surface models. A regression was also run at each surface point to compare differences between diagnostic groups and determine the association of clinical factors (chronicity, illness severity and medication) and psychosocial scores (quality of life and health status) with radial shrinkage. All statistical maps were directionally divided to indicate a positive or negative association; the maps shown are one-sided statistical maps in the hypothesised direction for each clinical measure (with lower volumes associated with poorer performance). Colour-coded probability values were assigned to the average surface models and their overall (corrected) significance was assessed using a permutation test. The $P$ values describe the significance of differences in radial distances between diagnostic groups or their association with clinical values. We used permutation tests to provide an overall significance value (a corrected $P$ value) for the statistical whole maps. The overall permutations $P$ was derived for left top, left bottom, right top and right bottom hippocampi. The primary threshold of $P=0.05$ was established for all statistical maps and the suprathreshold area was compared with its (non-parametric) null distribution based on randomised data. Correlations with clinical variables (length of illness, positive symptoms, negative symptoms, antipsychotic lifetime treatment) and psychosocial scores (MANSA, SF-36 physical component, SF-36 mental component) were separately corrected by applying Bonferroni correction.

\section{Results}

The demographic characteristics of the sample are listed in Table 1. All participants were White. All but one patient were receiving antipsychotic medication at the time of scanning: specifically, 25 patients were prescribed first-generation antipsychotic drugs (haloperidol $n=19$, fluphenazine $n=4$, zuclopenthixol $n=2$ ) and 41 patients were prescribed second-generation drugs (olanzapine $n=22$, clozapine $n=9$, risperidone $n=7$, quetiapine $n=3)$. Five of these patients were taking another antipsychotic at the time of imaging (clotiapine $n=2$, thioridazine $n=1$, quetiapine $n=1$, olanzapine $n=1$ ).

\section{Group difference maps}

We created 3D maps showing the mean level of hippocampal deflation in the schizophrenia group $v$. the control group. Separate maps were created for the left and right hippocampus. The results indicated no significant difference in either radial shrinkage or intracranial volume between the two groups (Table 1).

\section{Sociodemographic variables}

Significant associations were found in the maps for gender (left $P<0.001$; right $P<0.001$ ) and handedness (left $P<0.001$ ), with, as expected, a smaller hippocampus in women than in men and a larger left hippocampus in right-handed individuals. Next, we examined statistical effects of educational level in the schizophrenia group. Bilateral shape deflation was associated with lower levels of education in the schizophrenia group (left $P=0.001$; right $P<0.001)$ but not in the control group $(P>0.05)$; see online Fig. DS1.

\section{Clinical variables}

We created maps of associations between regional shape deflation and clinical scores. Bilateral hippocampal deflation was associated with greater duration of illness (left $P=0.01$, right $P=0.003$; after Bonferroni correction: left $P=0.04$, right $P=0.012)$ and higher levels of positive symptoms (left $P=0.05$, right $P=0.005$; after Bonferroni correction: left $P=0.20$, right $P=0.02$ ) and negative symptoms (left $P=0.01$, right $P=0.008$; after Bonferroni correction: left $P=0.04$, right $P=0.032$ ); see online Fig. DS2.

\begin{tabular}{|c|c|c|c|c|}
\hline & \multirow{2}{*}{$\begin{array}{l}\text { Control group } \\
\qquad(n=72)\end{array}$} & \multirow{2}{*}{$\begin{array}{l}\text { Schizophrenia group } \\
\qquad(n=67)\end{array}$} & \multicolumn{2}{|c|}{ Comparison } \\
\hline & & & Test & $P$ \\
\hline Age, years: mean (s.d.) & $39.3(11.0)$ & $39.4(12.1)$ & $t=0.15$ & 0.95 \\
\hline Gender, men/women: $n$ & $38 / 34$ & $44 / 23$ & $\chi^{2}=2.38$ & 0.12 \\
\hline Handedness, right/left: $n$ & $60 / 12$ & $55 / 12$ & $\chi^{2}=0.04$ & 0.85 \\
\hline Educational level, years: mean (s.d.) & $13.2(4.3)$ & $9.5(3.2)$ & $t=5.85$ & $<0.01$ \\
\hline Length of illness, years: mean (s.d.) & & $13.3(10.6)$ & & \\
\hline Antipsychotic lifetime treatment, years: mean (s.d.) & & $11.6(10.5)$ & & \\
\hline Total antipsychotic CPZ equivalent, mean (s.d.) & & $221.54(164.29)$ & & \\
\hline \multicolumn{5}{|l|}{ BPRS score, mean (s.d.) } \\
\hline Total score & & $45.4(18.3)$ & & \\
\hline Negative symptoms & & $11.8(4.7)$ & & \\
\hline Positive symptoms & & $11.7(6.3)$ & & \\
\hline MANSA score, mean (s.d.) & & $4.5(1.1)$ & & \\
\hline \multicolumn{5}{|l|}{ SF-36 score, mean (s.d.) } \\
\hline Physical component & & $49.2(7.9)$ & & \\
\hline Mental component & & $41.4(9.9)$ & & \\
\hline Intracranial volume, ml: mean (s.d.) & $1464.65(149.21)$ & $1467.20(172.49)$ & $t=-0.09$ & 0.93 \\
\hline
\end{tabular}


Controlling the analyses for intracranial volume, gender and handedness, negative symptoms and left hippocampal deflation showed a trend for significant correlation $(P=0.09)$. Finally, the effect of antipsychotic medication on the hippocampal size of the group with schizophrenia was examined, showing that more years of antipsychotic drug intake were associated with greater right hippocampal deflation (right $P=0.04$ ), which did not survive Bonferroni correction $(P=0.16)$. No significant correlation was observed between bilateral hippocampal region of interest volumes or intracranial volume and clinical measures (partial correlation analyses controlled for age, $P>0.05$, Bonferroni corrected).

\section{Psychosocial variables}

Examining generic health status with the SF-36, we found that lower levels of both physical health (left $P=0.01$, right $P=0.01$; after Bonferroni correction: left $P=0.03$, right $P=0.03$ ) and mental health (left $P=0.007$, right $P=0.01$; after Bonferroni correction: left $P=0.021$, right $P=0.03$ ) significantly correlated with bilateral deflation of the hippocampus. A similar association was found between a lower MANSA score and decreased hippocampal size (left $P=0.002$, right $P<0.001$; after Bonferroni correction: left $P=0.006$, right $P<0.003$ ); see online Fig. DS3. Controlling the analyses for intracranial volume, gender and handedness, SF-36 mental health scores and bilateral hippocampal deflation still correlated (right side $P=0.029$, left side $P=0.09$ ). The SF-36 and MANSA scores were not significantly associated with right and left hippocampal region of interest volumes or with intracranial volume (partial correlation analyses controlled for age, $P>0.05$, Bonferroni corrected).

\section{Discussion}

Using relatively novel methods for 3D mapping of the hippocampus, we did not detect significant hippocampal surface morphological differences at the group level in schizophrenia relative to agematched controls. This confirms our earlier study, ${ }^{17}$ and is in line with the somewhat controversial structural MRI literature summarised in the introduction, with some studies reporting hippocampal volume shrinkage and others reporting no abnormality. Notably, a preserved number and size of hippocampal neurons have been reported in schizophrenia. ${ }^{21}$ In addition, positron emission tomography and single photon emission computed tomography studies yielded a small effect size for hippocampal metabolism in differentiating schizophrenia and control groups, with an almost complete overlap (>92\%) between the two distributions. ${ }^{32}$ This suggests that hippocampal differences in schizophrenia, if present, might relate to local disturbances in cytoarchitectural organisation, ${ }^{33}$ with only partial or minimal overall effects on hippocampal morphology and metabolism. However, we investigated mainly patients with chronic disorder receiving long-term antipsychotic maintenance treatment, which may potentially have preserved or normalised the size of the hippocampus; decreased hippocampal volumes might be present in drug-naive people with a first episode of psychosis. ${ }^{34}$ Indeed, several human and animal studies suggest that some antipsychotic drugs may have neuroprotective effects on the hippocampus. ${ }^{35,36}$

Interestingly, with surface-based anatomical maps we found that greater hippocampal deflation was correlated with disease severity (duration of illness, positive and negative symptoms) and with poor social outcome identified in terms of lower levels of education, quality of life and health status. Consistent with this, prior imaging studies in schizophrenia found that smaller hippocampal volumes were associated with poorer premorbid adjustment, ${ }^{13}$ and with longer duration of illness. ${ }^{3,37}$ More severe positive symptoms have also been related to poorer hippocampal functioning. ${ }^{38}$ Furthermore, lower educational levels are associated with smaller grey-matter volumes (particularly in the temporal lobes) in healthy individuals, ${ }^{39}$ which might therefore have a greater negative impact on people with mental illness such as schizophrenia, as suggested by our findings. Our results are also supported by a study of patients with first-episode psychosis, in which smaller right hippocampal volumes were associated with worse clinical outcome at 3-year follow-up. ${ }^{40}$

\section{Outcomes in chronic illness}

Clinical outcomes may fluctuate during the first 10-15 years of schizophrenic illness and may not consistently relate to specific structural brain differences. ${ }^{41}$ Therefore it is extremely useful to investigate outcome measures in a cohort with chronic illness of long duration, as in our study. Prior imaging studies have explored structural markers of poor outcome in schizophrenia, differently defined based on admission rate, time spent in hospital, response to treatment, symptoms, self-care ability and general functioning. In particular, greater ventricular and lower cerebral (frontal) volumes have been reported, even with progressive changes over time in both chronic and first-episode disorder (see Bellani et al for review). ${ }^{22}$ Reduced volumes of the putamen and corpus callosum have also been reported by Mitelman et al in patients with very poor outcomes, identified as 'Kraepelinian'. ${ }^{42,43}$ These patients had, for the previous 5 years, been confined to hospital or needed continuous assistance, were unemployed and had no remission of symptoms. However, to the best of our knowledge, no prior report investigated specifically how hippocampal structure relates to specific psychosocial scales used to measure social functioning, as in our report. Therefore, it should be kept in mind that an impaired neuronal network, including in particular frontal cortex, putamen, corpus callosum and hippocampus, may be associated with a poor outcome in schizophrenia.

Our results suggest that illness severity and poor social outcome may reflect or promote poorer hippocampal neural plasticity in schizophrenia, with particular regional effects on the body of the hippocampal surface morphology localised predominantly on the right side, approximately including portions of the subiculum and of the cornu ammonis 1 subfields, although there was a scattered bilateral distributed impact. It should be considered that decreased environmental stimuli and chronic stress, which reduce the quality of life and the general health status of people with schizophrenia, are crucial factors that may contribute to affecting hippocampal plasticity. ${ }^{44,45}$ It is interesting to note that no significant correlation was found between clinical measures and hippocampal size considering the raw total volumes obtained by region of interest tracing, ${ }^{17}$ which we were able to observe using this $3 \mathrm{D}$ technique. This supports the strength of using advanced imaging and post-processing technique in analysing hippocampal morphology.

It is not yet fully understood whether hippocampal structural abnormalities partly reflect neurodegeneration in schizophrenia, but the hippocampus is one of the brain regions most vulnerable to neuronal loss. Diverse remodelling processes may change the morphology of hippocampus, such as retraction of dendrites, decreased neurogenesis and/or loss of glial cells, ${ }^{46}$ possibly in part due to altered glutamatergic neurotransmission. Also, oxidative DNA damage of the hippocampus has been found in elderly patients with schizophrenia and poor outcome. ${ }^{47}$ Hippocampal neurodegeneration may thus be an index of poor social outcome 
in people with chronic schizophrenia, particularly in a subset with neurodegenerative features.

\section{Limitations and future research}

Some major limitations of this study should be kept in mind. First, this was a cross-sectional study. To further characterise hippocampal volume based on social outcome, patients with schizophrenia should be studied longitudinally, ideally including drug-free individuals at the first outbreak. Second, neuropsychological and genetic assessments should be performed to determine how cognition and genes influence the statistical relationship between hippocampal volume and social functioning. Poor social functioning may in part be sustained by impaired cognitive abilities such as performance memory, learning and abstraction which relate to the hippocampus. Therefore, it would be intriguing to see whether cognitive decline or particular genetic polymorphisms such as BDNF and NRG1 promote changes in hippocampal morphology and associate with social outcome. Also, from 1.5 T MRI it was not possible to reliably resolve details of the anatomical subfields of the hippocampus, so we cannot argue with certainty which specific subregion consistently correlates with different clinical or psychosocial measures. Future 3D studies should therefore obtain anatomical fine definition of hippocampal subregions, ultimately trying to delineate whether regional shrinkage specifically links, for instance, with symptoms or poor social functioning. Finally, the ventricle system was not reconstructed in this study, so we cannot completely exclude that potential enlargement of the temporal horns of the lateral ventricles might relate to distortions in surrounding anatomy, including the hippocampus. However, considering that no significant difference in hippocampal volume was found between the patient and control groups, we assume that possible effects of ventricular enlargement did not take place in this case.

In conclusion, hippocampal deflation may be a structural sign of poor clinical outcome and social functioning in schizophrenia, helping to identify a subgroup of patients who might need specific treatment. Classification of patients with hippocampal abnormalities and clinical and social impairments should, however, be based on specific criteria of illness severity and poor functioning, which might then be of clinical relevance. This may indeed assist in determining patients who would benefit from adequate therapeutic management such as psychosocial stimulation, cognitive rehabilitation or physical exercise. Such therapeutic strategies might potentially preserve or normalise hippocampal size, which could also be monitored to study the effects of clinical interventions, ultimately leading to better clinical and social outcomes.

Paolo Brambilla, MD, PhD, Department of Experimental and Clinical Medical Sciences (DISM), InterUniversity Centre for Behavioural Neurosciences, University of Udine, and Scientific Institute IRCCS 'E. Medea', Udine, Italy; Cinzia Perlini, PsychD, PhD, Department of Public Health and Community Medicine, Section of Psychiatry and Section of Clinical Psychology, InterUniversity Centre for Behavioural Neurosciences, University of Verona, Italy; Priya Rajagopalan, MPH, MBBS, Neurosciences, University of Verona, Italy; Priya Rajagopalan, MPH, MBBS,
Priyanka Saharan, BS, Laboratory of Neuro Imaging (LONI), David Geffen School of Medicine at UCLA, Department of Neurology, Los Angeles, California, USA; Gianluca Rambaldelli, MS, Marcella Bellani, MD, PhD, Nicola Dusi, MD, Department of Public Health and Community Medicine, Section of Psychiatry and Section of Clinical Psychology, InterUniversity Centre for Behavioural Neurosciences, University of Verona, Italy; Roberto Cerini, MD, Roberto Pozzi Mucelli, MD, Department of Pathology and Diagnosis, Section of Radiology, G.B. Rossi Hospital, University of Verona, Italy; Michele Tansella, MD, Department of Public Health University of Verona, Italy; Michele Tansella, MD, Department of Public Health
and Community Medicine, Section of Psychiatry and Section of Clinical Psychology, InterUniversity Centre for Behavioural Neurosciences, University of Verona, Italy; Paul M. Thompson, PhD, LONI, David Geffen School of Medicine at UCLA, Department of Neurology, Los Angeles, California, USA

Correspondence: Dr Paolo Brambilla, Clinica Psichiatrica, AOU, P. Ie S. Maria della Misericordia 15, 33100 Udine, Italy. Email: paolo.brambilla@uniud.it

First received 10 Nov 2011, final revision 27 May 2012, accepted 18 oct 2012

\section{Funding}

P.B. was partly supported by grants from the American Psychiatric Institute for Research and Education, the Italian Ministry for University and Research and the Italian Ministry of Health (IRCCS 'E. Medea'). P.R., P.S. and P.M.T. were supported by National Institute of Health grants R01 EB008281, P41 RR013642, R01 AG020098 and R01 HD050735.

\section{References}

1 Sim K, DeWitt I, Ditman T, Zalesak M, Greenhouse I, Goff D, et al. Hippocampal and parahippocampal volumes in schizophrenia: a structural MRI study. Schizophr Bull 2006; 32: 332-40.

2 Narr KL, Thompson PM, Szeszko P, Robinson D, Jang S, Woods RP, et al. Regional specificity of hippocampal volume reductions in first-episode schizophrenia. Neuroimage 2004; 21: 1563-75.

3 Chakos MH, Schobel SA, Gu H, Gerig G, Bradford D, Charles C, et al. Duration of illness and treatment effects on hippocampal volume in male patients with schizophrenia. Br J Psychiatry 2005; 186: 26-31.

4 Steen RG, Mull C, McClure R, Hamer RM, Lieberman JA. Brain volume in firstepisode schizophrenia. Systematic review and meta-analysis of magnetic resonance imaging studies. Br J Psychiatry 2006; 188: 510-8.

5 Steen RG, Hamer RM, Lieberman JA. Measurement of brain metabolites by $1 \mathrm{H}$ magnetic resonance spectroscopy in patients with schizophrenia: a systematic review and meta-analysis. Neuropsychopharmacology 2005; 30 : 1949-62.

6 Wang L, Mamah D, Harms MP, Karnik M, Price JL, Gado MH, et al. Progressive deformation of deep brain nuclei and hippocampal-amygdala formation in schizophrenia. Biol Psychiatry 2008; 64: 1060-8.

7 Boos HB, Aleman A, Cahn W, Hulshoff Pol H, Kahn RS. Brain volumes in relatives of patients with schizophrenia: a meta-analysis. Arch Gen Psychiatry 2007; 64: 297-304.

8 Velakoulis D, Wood SJ, Wong MT, McGorry PD, Yung A, Phillips L, et al. Hippocampal and amygdala volumes according to psychosis stage and diagnosis: a magnetic resonance imaging study of chronic schizophrenia, first-episode psychosis, and ultra-high-risk individuals. Arch Gen Psychiatry 2006; 63: 139-49.

9 Ellison-Wright I, Glahn DC, Laird AR, Thelen SM, Bullmore E. The anatomy of first-episode and chronic schizophrenia: an anatomical likelihood estimation meta-analysis. Am J Psychiatry 2008; 165: 1015-23.

10 Phillips $L$, Yung AR, Yuen HP, Pantelis C, McGorry PD. Prediction and prevention of transition to psychosis in young people at incipient risk for schizophrenia. Am J Med Genet 2002; 114: 929-37.

11 Staal WG, Hulshoff Pol HE, Schnack HG, Hoogendoorn ML, Jellema K, Kahn RS. Structural brain abnormalities in patients with schizophrenia and their healthy siblings. Am J Psychiatry 2000; 157: 416-21.

12 Sanfilipo $\mathrm{M}$, Lafargue $\mathrm{T}$, Rusinek $\mathrm{H}$, Arena $\mathrm{L}$, Loneragan $\mathrm{C}$, Lautin $\mathrm{A}$, et al. Cognitive performance in schizophrenia: relationship to regional brain volumes and psychiatric symptoms. Psychiatry Res 2002; 116: 1-23.

13 Smith GN, Lang DJ, Kopala LC, Lapointe JS, Falkai P, Honer WG. Developmental abnormalities of the hippocampus in first-episode schizophrenia. Biol Psychiatry 2003; 53: 555-61.

14 Frazier JA, Hodge SM, Breeze JL, Giuliano AJ, Terry JE, Moore CM, et al. Diagnostic and sex effects on limbic volumes in early-onset bipolar disorder and schizophrenia. Schizophr Bull 2008; 34: 37-46.

15 Glahn DC, Laird AR, Ellison-Wright I, Thelen SM, Robinson JL, Lancaster JL, et al. Meta-analysis of gray matter anomalies in schizophrenia: application of anatomic likelihood estimation and network analysis. Biol Psychiatry 2008; 64: 774-81.

16 Nelson MD, Saykin AJ, Flashman LA, Riordan HJ. Hippocampal volume reduction in schizophrenia as assessed by magnetic resonance imaging: a meta-analytic study. Arch Gen Psychiatry 1998; 55: 433-40.

17 Baiano M, Perlini C, Rambaldelli G, Cerini R, Dusi N, Bellani M, et al Decreased entorhinal cortex volumes in schizophrenia. Schizophr Res 2008; 102: $171-80$.

18 Jeste DV, Lohr JB. Hippocampal pathologic findings in schizophrenia. A morphometric study. Arch Gen Psychiatry 1989; 46: 1019-24.

19 Heckers S, Stone D, Walsh J, Shick J, Koul P, Benes FM. Differential hippocampal expression of glutamic acid decarboxylase 65 and 67 messenger RNA in bipolar disorder and schizophrenia. Arch Gen Psychiatry 2002; 59: 521-9.

20 Hurlemann R, Tepest R, Maier W, Falkai P, Vogeley K. Intact hippocampal gray matter in schizophrenia as revealed by automatized image analysis postmortem. Anat Embryol (Berl) 2005; 210: 513-7. 
21 Walker MA, Highley JR, Esiri MM, McDonald B, Roberts HC, Evans SP, et al Estimated neuronal populations and volumes of the hippocampus and its subfields in schizophrenia. Am J Psychiatry 2002; 159: 821-8.

22 Bellani M, Dusi N, Brambilla P. Longitudinal imaging studies in schizophrenia: the relationship between brain morphology and outcome measures. Epidemiol Psichiatr Soc 2010; 19: 207-10.

23 Thompson PM, Hayashi KM, De Zubicaray GI, Janke AL, Rose SE, Semple J, et al. Mapping hippocampal and ventricular change in Alzheimer disease. Neuroimage 2004; 22: 1754-66.

24 American Psychiatric Association. Diagnostic and Statistical Manual of Mental Disorders (4th edn) (DSM-IV). APA, 1994.

25 Wing JK (ed). WHO Schedules for Clinical Assessment in Neuropsychiatry. World Health Organization, 1992.

26 Tansella M, Nardini M. SCAN - Schede di Valurazione Clinica in Neuropsichatria. II Pensiero Scientifico Editore, 1996.

27 Overall JE, Gorham DL. The Brief Psychiatric Rating Scale. Psychol Rep 1962 10: 789-812.

28 Ware JE, Sherbourne CD. The MOS 36-item short-form health survey (SF-36) Med Care 1992; 30: 473-83.

29 Priebe S, Huxley $P$, Knight S, Evans S. Application and results of the Manchester Short Assessment of Quality of Life (MANSA). Int J Soc Psychiatry 1999; 45: 7-12.

30 First MB, Spitzer RL, Gibbon M, Williams JBW. The Structured Clinical Interview for DSM-IV Axis I Disorders, Non-Patient Version (SCID-I/NP). Biometrics Research, New York State Psychiatric Institute, 1996.

31 Brambilla $P$, Harenski K, Nicoletti M, Sassi RB, Mallinger AG, Frank E, et al. MRI investigation of temporal lobe structures in bipolar patients. J Psychiat Res 2003; 37: 287-95.

32 Davidson LL, Heinrichs RW. Quantification of frontal and temporal lobe brain imaging findings in schizophrenia: a meta-analysis. Psychiatry Res 2003; 122 69-87.

33 Heckers S, Konradi C. Hippocampal neurons in schizophrenia. J Neural Transm 2002; 109: 891-905.

34 Vita A, De Peri L, Silenzi C, Dieci M. Brain morphology in first-episode schizophrenia: a meta-analysis of quantitative magnetic resonance imaging studies. Schizophr Res 2006; 82: 75-88.

35 Pillai A, Mahadik SP. Differential effects of haloperidol and olanzapine on levels of vascular endothelial growth factor and angiogenesis in rat hippocampus. Schizophr Res 2006; 87: 48-59.
36 Bearden $C E$, Thompson $\mathrm{PM}$, Avedissian $\mathrm{C}$, Klunder $\mathrm{AD}$, Nicoletti $\mathrm{M}$, Dierschke $\mathrm{N}$, et al. Altered hippocampal morphology in unmedicated patients with major depressive illness. ASN Neuro 2009; 1: e00020.

37 Velakoulis D, Pantelis C, McGorry PD, Dudgeon P, Brewer W, Cook M, et al. Hippocampal volume in first-episode psychoses and chronic schizophrenia: a high-resolution magnetic resonance imaging study. Arch Gen Psychiatry 1999; 56: 133-41.

38 Goghari VM, Sponheim SR, MacDonald AW. The functional neuroanatomy of symptom dimensions in schizophrenia: a qualitative and quantitative review of a persistent question. Neurosci Biobehav Rev 2010; 34: 468-86.

39 Ho AJ, Raji CA, Becker JT, Lopez OL, Kuller LH, Hua X, et al. The effects of physical activity, education, and body mass index on the aging brain. Hum Brain Mapp 2011; 32: 1371-82.

40 De Castro-Manglano P, Mechelli A, Soutullo C, Landecho I, Gimenez-Amaya JM, Ortuno $\mathrm{F}$, et al. Structural brain abnormalities in first-episode psychosis: differences between affective psychoses and schizophrenia and relationship to clinical outcome. Bipolar Disord 2011; 13: 545-55.

41 Staal WG, Hulshoff Pol HE, Kahn RS. Outcome of schizophrenia in relation to brain abnormalities. Schizophr Bull 1999; 25: 337-48.

42 Mitelman SA, Canfield EL, Chu KW, Brickman AM, Shihabuddin L, Hazlett EA, et al. Poor outcome in chronic schizophrenia is associated with progressive loss of volume of the putamen. Schizophr Res 2009; 113: 241-5.

43 Mitelman SA, Nikiforova YK, Canfield EL, Hazlett EA, Brickman AM, Shihabuddin L, et al. A longitudinal study of the corpus callosum in chronic schizophrenia. Schizophr Res 2009; 114: 144-53.

44 Zhang L, Zhang J, Sun $\mathrm{H}$, Liu H, Yang Y, Yao Z. Exposure to enriched environment restores the mRNA expression of mineralocorticoid and glucocorticoid receptors in the hippocampus and ameliorates depressive-like symptoms in chronically stressed rats. Curr Neurovasc Res 2011; 8: 286-93.

45 Hu XL, Bergstrom SA, Brink M, Ronnback A, Dahlqvist P. Enriched environment increases spinophilin mRNA expression and spinophilin immunoreactive dendritic spines in hippocampus and cortex. Neurosci Lett 2011; 476: 79-83.

46 Martinez-Tellez RI, Hernandez-Torres E, Gamboa C, Flores G. Prenatal stress alters spine density and dendritic length of nucleus accumbens and hippocampus neurons in rat offspring. Synapse 2009; 63: 794-804.

47 Nishioka N, Arnold SE. Evidence for oxidative DNA damage in the hippocampus of elderly patients with chronic schizophrenia. Am J Geriatr Psychiatry 2004; 12: 167-75. 